J. Sustain. Wireless Syst.

Vol.02/ No. 2

Pages: $92-99$

http://irojournals.com/irosws/

DOI: https://doi.org/10.36548/jsws.2020.2.005

\title{
Initial Access through Beamforming in mmWave
}

G. Christina

Assistant Professor,

Department of Electronics and Communication Engineering,

Coimbatore Institute of Technology,

Coimbatore, India

E-mail: gchristina@cit.edu.in

\begin{abstract}
Future Wi-Fi, 5G Cellular and millimetre-wave (mmWave) will depend on highly directional links in order to prevail over exuberant path loss experienced in the different bands of frequency. However, in order to establish these type of links, the receiver and transmitter need mutual discovery which will result in high energy consumption and large latency. The proposed work deals with reduction of energy consumption and latency significantly with the help of a fully digital front-end. The digital beamformer will receive the spatial samples within a shot, from all directions. However, in analog front-ends, sampling is allowed for beamforming in one particular direction at a time resulting in the time period in which the mobile is "on" for longer. This will result in an increase in energy consumption by more than four times for the analog front-end when compared with digital front-ends, taking into consideration the antenna arrays' size. However, from the power consumption point of view, using a fully digital beamforming post beam discovery is not recommended. Hence in order to overcome this drawback, a digital beamformer coupled with a 4-bit A-D convertor with low resolution is proposed. The use of low resolution will decrease the power consumption such that it is in the same zone as that of analog beam forming while it is possible to make use of the fully digital beamforming spatial multiplexing capabilities resulting in improved energy efficiency and reduced discovery latency.
\end{abstract}

Keywords: Digital beamformer, analog beam former, path loss, directional links

\section{Introduction}

In general most wireless communication that exists today will make use of the electromagnetic spectrum that lies below $6 \mathrm{GHz}$ which is the bandwidth allocated for public, military, civilian, commercial safety and experimental use [1]. However, the demand for the devices and services used over this bandwidth has resulted in a lot of discrepancy in terms of degrees of freedom (DoF) and availability [2-4]. This will result in reduced quality of service and lower data rates. Moreover, as the use of Device to device communication and Internet of Things and Device increases, it will further crush the available spectrum with traffic and overloaded networks. Hence the only possible solution is to increase the DoFs in the upcoming wireless systems. mmWave makes use of many DoFs by increasing the bandwidth availability using the addition of unlicensed spectrum. But, in [5] Friis' law explains that there is possibility of high isotropic path loss when using mmWaves to transmit signals. This will result in the range of communication to become smaller when compared to other $6 \mathrm{Ghz}$ systems. Moreover, the mmWave carriers characteristics [6] similar to visible light [7]. mmWaves make use of beamforming in order to overcome their disadvantage of high penetration loss. Hence an array of multiple antenna 
J. Sustain. Wireless Syst.

Vol.02/ No. 2

Pages: $92-99$

http://irojournals.com/irosws/

DOI: https://doi.org/10.36548/jsws.2020.2.005

elements are used to avoid environmental obstacles by guiding the signal energy in the right path. The drawback is that the use of directional links through beamforming gives rise to new challenges that are unknown in wireless systems. Here the receiver and transmitter must initially discover each other before commencing the communication of data which is commonly referred to as initial access [8]. This is generally easy to perform in 6-GHz bands. However since mmWave imposes high path loss, it is not wise to use the same methodology. Hence mutual discovery in a directional manner is recommended for these signals. Hence when arrays of multiple antenna resulting in narrow beams are formed, it is possible to ensure that the directional discovery phase lasts longer. However, energy burning is high during the search for the base-station. In the proposed work, we show that a reduction in this energy consumed is possible when the user end uses a low-resolution fully digital front-end. This is because, when compared to analog front-end, the digital front-end beam forming will significantly decrease the delay or discovery latency[9-10]. This ensures that the user is 'on' for only a short period of time, resulting in saving energy. The main aim of this work is towards directional discovery in initial access, addressing the issues of energy consumption and latency in mmWave system. Based on the results observed, we can summarize two key takeaways from this work which includes:

1. Low power consumption and low latency for data transmission and control signalling is possible when using low resolution ADCs in fully digital front-ends [11].

2. When compared to analog beamforming, digital beamforming will result in reduced energy consumption and latency during initial discover process.

\section{Beam Discovery Using Beam Sweep}

Let us take a transmitter-receiver pair that is functioning in the mmWave bandwidth. It is necessary that they first discover each other in order to communicate with the help of directional links before data communication takes place. One way of dealing with this problem is by dividing the spatial domain surrounding the receiver (or transmitter) into a number of sectors [12-13] which are overlapping such that event sector represents a receiver or transmitter beam. A beamspace is defined by all possible combinations of the transmitter-receiver beam pairs. The beams are made narrow by increasing the antennas count thereby increasing gain of beamforming. However, this will also increase the size of beamspace. This is one of the basic problems faced by mmWave systems. Though the narrow beams obtained will aid in link budget [14], it is difficult in terms of beam discovery. A standalone communication model is assumed in this proposed work, such that the receiver and transmitter do not require any information regarding position and timing. Based on this assumption, there are two main approaches for beam selection/ discovery

1. In one approach listening on the channel begins on the receiver side taking into consideration the widest beam possible and later on converges to a narrower beam in a step-by-step manner. This approach is known as hierarchical search.

2. The other approach requires the receiver and transmitter to search exhaustively over the entire beamspace with the help of received power measurement for the beam pair. 
J. Sustain. Wireless Syst.

Vol.02/ No. 2

Pages: $92-99$

http://irojournals.com/irosws/

DOI: https://doi.org/10.36548/jsws.2020.2.005

Both the approaches will require a synchronization signal which is known. This signal is transmitted periodically and based on the beamspace direction, the receiver will diagnose the areas where there is stronger incoming signal. Because of the simplicity of beamsweep, mmWave standards for cellular and WiFi have adopted this technology over hierarchical search. We have also followed the beamsweep paradigm in the proposed posed.

\section{Beam Discovery}

In the beginning, initial access is accomplished through message exchange between the user equipment and gNB such that the user first finds a gNB that is serving and synchronizes to it. From the gNB, the physical call identity is identified and this is used to send back the user's own ID in order to complete the process and attach to the cell. The proposed work concentrates on cell and beam discovery as it is very crucial in terms of energy consumption and detection delay. The surrounding area is swept with at the time interval of $\mathrm{T}=20 \mathrm{~ms}$ at the $\mathrm{gNB}$ side. To be more precise, for every interval $\mathrm{T}_{\mathrm{ss}}=5 \mathrm{~ms}, 4 \mathrm{IFDM}$ symbols are transmitted in the direction of $\mathrm{N}_{\mathrm{Tx}}$. Here every block is known as an SS block and this transmission is known as synchronization signal (SS) burst. Every SS block is made up of Physical broadcast channel (PBCH), secondary synchronization signal (SSS) and primary synchronization signal (SSS). The physical ID of the cell is made up of PSS and SSS, taken together. Based on this, a total of 1008 unique IDs are created and about 127 sub carriers in frequency are taken by up by these signals, resulting in a total of 240 sub-carriers on including $\mathrm{PBCH}$.

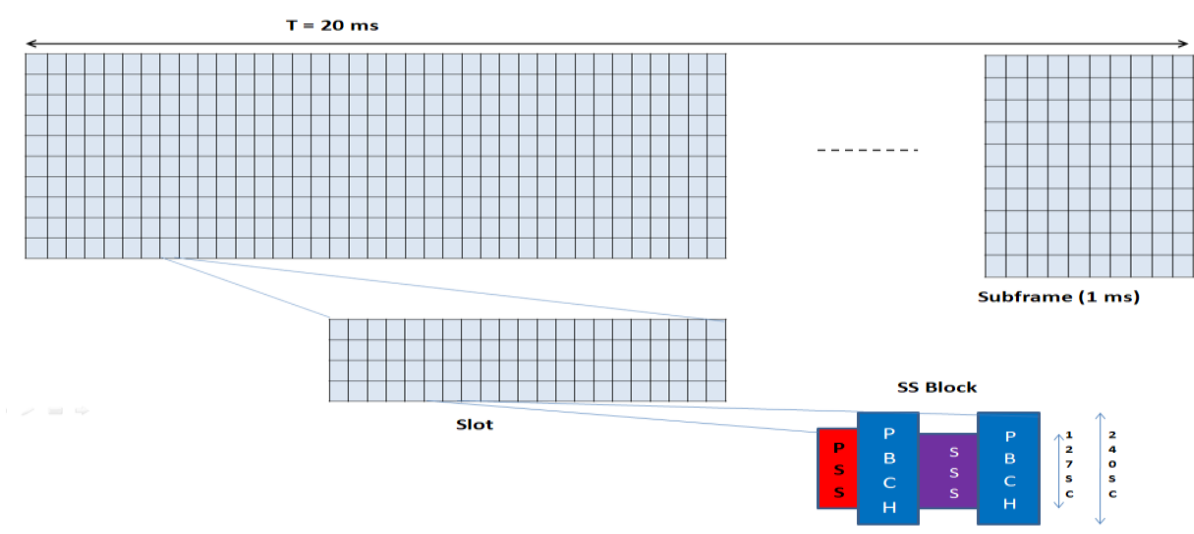

Fig.1. Frame Structure with frames divided into 8 slots and 14 OFDM symbols

Here the figure Fig.2. represents the SS Block inside the NR frame, in terms of time-frequency. Similarly Fig.3. represents the presence of many SS blocks inside the SS burst. A total of 32 blocks are present within the SS burst for mmWave bands. We assume that the SS blocks are transmitted with hybrid beamforming and simultaneously in different directions. There are about 64 non-lapping directions which can be supported by the mmWave gNB. The SS burst is further searched for by 
J. Sustain. Wireless Syst.

Vol.02/ No. 2

Pages: $92-99$

http://irojournals.com/irosws/

DOI: https://doi.org/10.36548/jsws.2020.2.005

sweeping $\mathrm{N}_{\mathrm{Rx}}$ direction for a $\mathrm{gNB}$ beamsweeping of $\mathrm{N}_{\mathrm{Tx}}=8$. The signals used are predefined and known in order to find the 336 SSS sequences and 3 PSS sequences that have been transmitted. When the PSS signal is discovered, it is possible to find the optimal direction as the structure of SS block is already known. Next, the user equipment will work towards finding SSS which is located inside the same SS block as represented in Fig.2. This is why detecting PSS is fixed to be the most vital part of beamsweeping that can be used to find the rest of the initial access steps. When An analog beam is used, it is possible for the gNB to probe in only one direction. However, the SS blocks are sent by the gNB in 2 different directions at the same time.

$$
\mathrm{L}=\left(\mathrm{N}_{\mathrm{TX}} \times \mathrm{N}_{\mathrm{Rx}}\right)
$$

where $\mathrm{L}$ is the size of beamspace that is present around the user equipment and gNB.

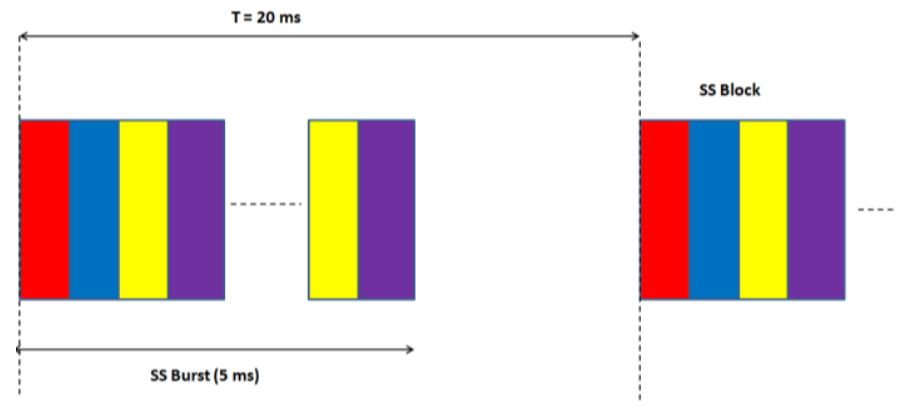

Fig.2. SS Burst

\section{Results and Discussion}

Fig.3. represents the energy consumption of analog and digital front-ends with $\mathrm{N}_{\mathrm{Rx}}$ fixed at 16 for the user equipment. However, the variation between digital and analog is very large. In fact, it is noticed that the energy consumed by the analog version mountains over the low-resolution and high-resolution digital version. When the power consumption of digital beamforming was analyzed, the resultant graph showed that it was about 6 times the consumption of analog beamforming. It also shows that during beamsweeping, the level of burning of analog beamforming frontend is about 4 times more than that of the digital front end. But, once the directional link is established, it is expected that the analog front end burns less than that of digital frontend in the data communication phase, resulting in the need for lowresolution digital front-end. The energy consumption difference is significantly small duing beam discovery. 
J. Sustain. Wireless Syst.

Vol.02/ No. 2

Pages: $92-99$

http://irojournals.com/irosws/

DOI: https://doi.org/10.36548/jsws.2020.2.005

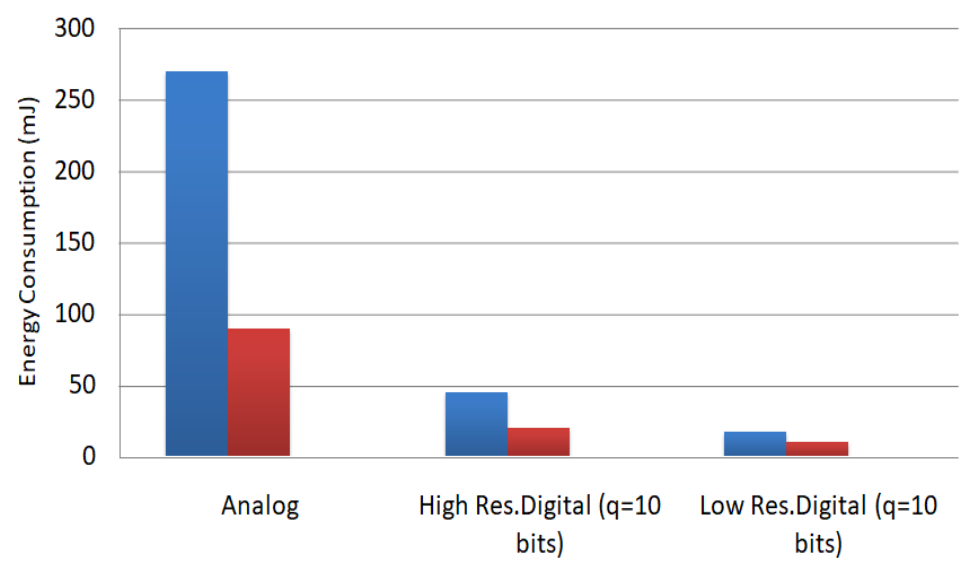

Fig.3. Energy Consumption

It is also necessary to investigate the receiver side antenna numbers. At the initial stage of beamsweeping phase, it is important to analyze how the energy consumption scales with respect to the antenna array size. This will give a detailed report on how energy consumption is impacted by the different sizes of beamspace.

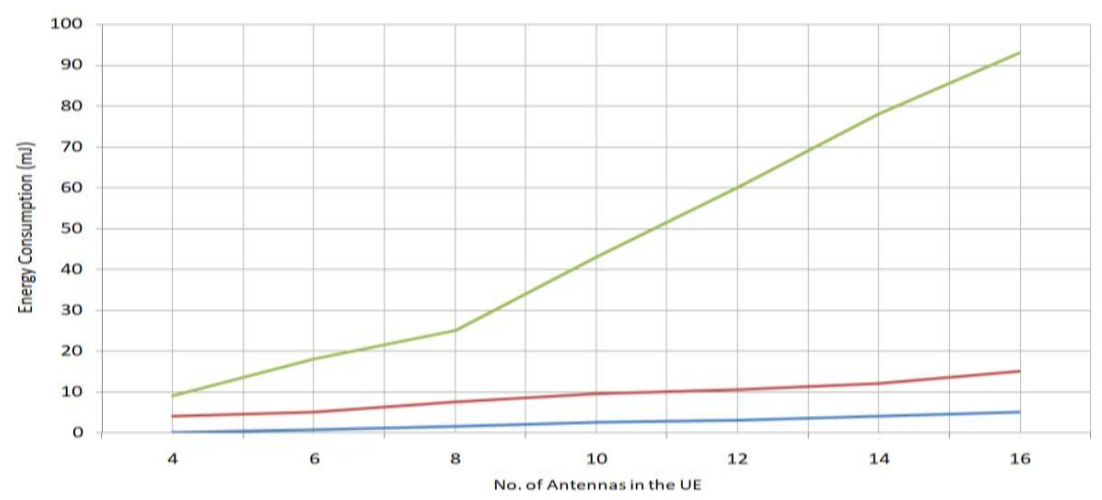

Fig.4. Median User Equipment

To figure out the effect of antenna numbers, different user equipment configurations are used such as 2D array of size 16, 8 and 4 as shown in Fig.4. and Fig.5.

Based on the two figures, there are some interesting things to be considered: 
J. Sustain. Wireless Syst.

Vol.02/ No. 2

Pages: $92-99$

http://irojournals.com/irosws/

DOI: https://doi.org/10.36548/jsws.2020.2.005

- Even though the use of lesser number of antennas in order to decrease the energy consumption, and more amount of energy is utilized by the analog beamforming when compared to the digital front-ends.

- At the user equipment, when many antennas are used, the gap between digital and analog beamforming will increase.

This is mainly due to the following reason: analog beamforming will see less number of directions at every interval, SNR is low after BF and the number of antennas is low.

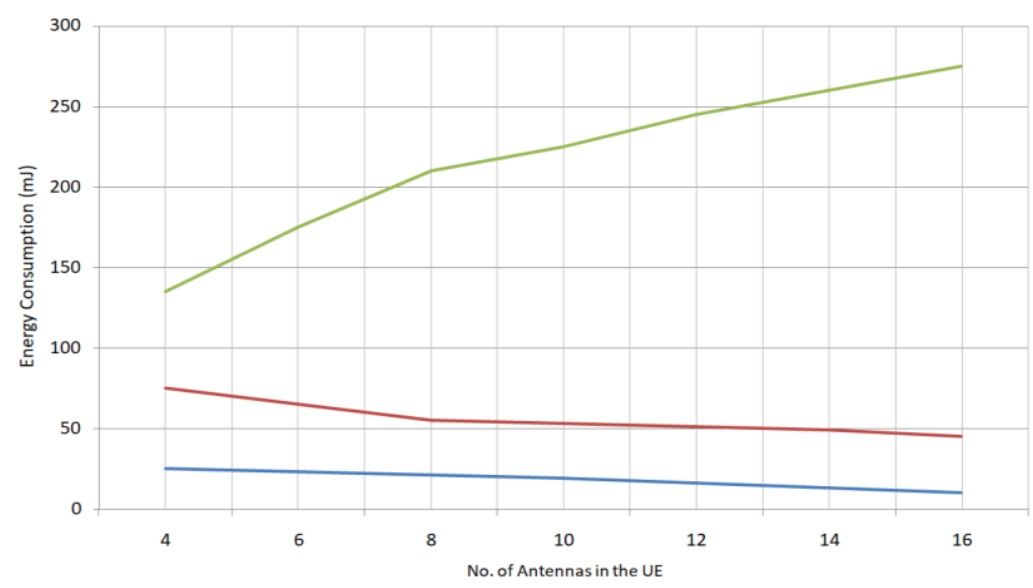

Fig.5. Cell Edge User Equipment

\section{Conclusion}

There are two important challenges that are addressed by the mmWave communication systems. They are energy consumption and beam discovery. In the proposed paper, we have explained how the two factors relate closely to each other. This is especially shown in the analog beamforming which shows an increase in both energy consumption and discover delay. Hence it is not suitable for low energy consumption. However lower delay and energy consumption is possible when digital beamforming is used in the place of analog. Based on the Signal to Noise Ratio, joint flexible scheduling of the resources of frequency and multi-stream communication is allowed when digital beamforming is used. It is found that this is very useful for transmitting small data packets. Due to the less frequency of paging and texting, a more aggressive usage of sleep mode is possible for digital use. The future research might involve studying of the architecture of beamforming in various layers like TCP/IP and MAC. Hence it is found that the realization of mmWave cellular system using low-resolution beamforming will result in low delay while giving way for scheduling flexibility. 
J. Sustain. Wireless Syst.

Vol.02/ No. 2

Pages: $92-99$

http://irojournals.com/irosws/

DOI: https://doi.org/10.36548/jsws.2020.2.005

\section{References}

[1] 3GPP: NR-Physical Layer Procedures for Control. TS 38.213 (release 15) (2018)

[2] 3GPP: NR-Radio Resource Control (RRC) Protocol Specification. TS 38.331 (release 15) (2018)

[3] Abbas, W.B., Zorzi, M.: Context information based initial cell search for millimeter wave $5 \mathrm{~g}$ cellular networks. In: 2016 European Conference on Networks and Communications (EuCNC), pp. 111-116 (2016)

[4] Adabi, E., Heydari, B., Bohsali, M., Niknejad, A.M.: $30 \mathrm{GHz}$ CMOS low noise amplifier. In: Proc. IEEE RFIC Symp., pp. 625-628 (2007)

[5] Akdeniz, M., Liu, Y., Samimi, M., Sun, S., Rangan, S., Rappaport, T., Erkip, E.: Millimeter wave channel modeling and cellular capacity evaluation. IEEE J. Sel. Areas Commun. 32(6), 1164-1179 (2014)

[6] Alkhateeb, A., Nam, Y., Rahman, M.S., Zhang, J., Heath, R.W.: Initial beam association in millimeter wave cellular systems: Analysis and design insights. IEEE Trans. on Wireless Commun. 16(5), 2807-2821 (2017)

[7] Azar, Y., Wong, G.N., Wang, K., Mayzus, R., Schulz, J.K., Zhao, H., Gutierrez, F., Hwang, D., Rappaport, T.S.: $28 \mathrm{GHz}$ propagation measurements for outdoor cellular communications using steerable beam antennas in New York City. In: Proc. IEEE ICC (2013)

[8] Barati, C.N., Hosseini, S., Rangan, S., Liu, P., Korakis, T., Panwar, S., Rappaport, T.S.: Directional cell discovery in millimeter wave cellular networks. IEEE Trans. Wireless Commun. 14(12), 6664 - 6678 (2015) [12] Barati, C.N., Hosseini, S.A., Mezzavilla, M., Korakis, T., Panwar, S.S., Rangan, S., Zorzi, M.: Initial access in millimeter wave cellular systems. IEEE Trans. Wireless Commun 15(12), 7926-7940 (2016)

[9] Booth, M.B., Suresh, V., Michelusi, N., Love, D.J.: Multi-armed bandit beam alignment and tracking for mobile millimeter wave communications. IEEE Communications Letters 23(7), 1244-1248 (2019)

[10] Chiu, S., Ronquillo, N., Javidi, T.: Active learning and csi acquisition for mmwave initial alignment. IEEE J. Sel. Areas Commun. 37(11), 2474-2489 (2019)

[11] Devoti, F., Filippini, I., Capone, A.: Mm-wave initial access: A context information overview. In: 2018 IEEE 19th International Symposium on "A World of Wireless, Mobile and Multimedia Networks" (WoWMoM), pp. 1-9 (2018) 
J. Sustain. Wireless Syst.

Vol.02/ No. 2

Pages: $92-99$

http://irojournals.com/irosws/

DOI: https://doi.org/10.36548/jsws.2020.2.005

[12] Dutta, S., Barati, C.N., Ramirez, D., Dhananjay, A., Buckwalter, J.F., Rangan, S.: A case for digital beamforming at mmwave. IEEE Trans. Wireless Commun. pp. 1-1 (2019)

[13] Haoxiang, W., \& Smys, S. (2020). Soft Computing Strategies for Optimized Route Selection in Wireless Sensor Network. Journal of Soft Computing Paradigm (JSCP), 2(01), 1-12.

[14] Raj, J. S. (2020). Machine Learning Based Resourceful Clustering With Load Optimization for Wireless Sensor Networks. Journal of Ubiquitous Computing and Communication Technologies (UCCT), 2(01), 29-38.

\section{Authors Biography}

G. Christina works as Assistant Professor in the Department of Electronics and Communication Engineering in Coimbatore Institute of Technology in Coimbatore, Tamil Nadu, India, her area of research includes Sustainable information systems, Wireless Networks, Internet of Things, Computer Networks, Mobile Communication, Software Defined Wireless communication systems, Cyber Physical Systems, Green Data Centers, Cognitive principles and techniques. 